\title{
Checklist of the benthic marine macroalgae from Algeria. I. Phaeophyceae
}

\author{
Nora Ould-Ahmed ${ }^{1 *}$, Amelia Gómez Garreta², María Antonia Ribera Siguan² \& Nadia Bouguedoura ${ }^{3}$ \\ ${ }^{1}$ Ecole Nationale Supérieure des Sciences de la Mer et de l'Aménagement du Littoral (ENSSMAL), Campus Universitaire de Dely-Îlbrahim, \\ B.P. 19, Bois des cars, 16320 Alger, Algeria; noraouldahmed@yahoo.fr \\ ${ }^{2}$ Laboratori de Botànica, Facultat de Farmàcia, Universitat de Barcelona, Av. Joan XXIII s/n, E-08028 Barcelona, Spain; \\ ameliagomez@ub.edu; riberasiguan@ub.edu \\ ${ }^{3}$ Université des Sciences et Technologie Houari Boumedienne, Biologie et Physiologie, B.P, 31 El Alia Bab Ezzouar Algeries (Algeria); \\ nadiaboug@gmail.com
}

\begin{abstract}
Ould-Ahmed, N., Gómez Garreta, A., Ribera Siguan, M.A. \& Bouguedoura, N. 2013. Checklist of the benthic marine macroalgae from Algeria. I. Phaeophyceae. Anales Jard. Bot. Madrid 70(2): 136-143.

The seaweed diversity of the Mediterranean is still not completely known, especially in some areas of its African coasts. As an effort to complete a more detailed catalogue to fill such gap, an updated checklist of the brown seaweeds (Phaeophyceae) from Algeria, based on updated literature records, is provided using as starting point the checklist of PerretBoudouresque \& Seridi published in 1989. As a result, the total number of taxa at specific and infraspecific levels accepted for Algeria, under current taxonomy and nomenclature, is 93.
\end{abstract}

Keywords: Algerian coast, brown algae, catalogue, Phaeophyceae, seaweeds

\section{INTRODUCTION}

The flora of seaweeds on the Algerian coast has been well studied by classical French algologists, among them are Montagne (1838, 1846-1849, 1856), Debray (1893, 1897), Feldmann \& Feldmann-Mazoyer (1931-1961, see Perret-Boudouresque \& Seridi, 1989). In 1989, Perret-Boudouresque \& Seridi published a checklist of the benthic marine macroalgae which compiled all the information known on this area up to that moment. In 1992, a group of Mediterranean algologists provided the first part of the checklist of the Mediterranean seaweeds, corresponding to the Phaeophyceae (Ribera \& al., 1992). In that paper, a taxonomical and nomenclatural critical revision of all the Mediterranean brown seaweeds was carried out. Concerning the Algerian flora, the paper by PerretBoudouresque \& Seridi (1989) was used as reference. In the two last decades, some authors have continued working on the marine flora from Algeria (Ould Ahmed \& al., 1995; Ould Ahmed, 2005; Ould Ahmed \& Meinesz, 1998, 2007; Seridi \& al., 2007), In addition, there is more information which is often difficult to consult, since it is mostly included in unpublished papers, such as PhD theses (Seridi, 1990, 2007; KadariMéziane, 1994; Ould-Ahmed, 1994, 2013). Báez \& al. (2004, 2005a, b) used the data of the Algerian Cystoseira species compiled in Ribera \& al. (1992) to carry out a geographical analysis of species richness in the Mediterranean sea, without contributing any new data.

\section{Resumen}

Ould-Ahmed, N., Gómez Garreta, A., Ribera Siguan, M.A. \& Bouguedoura, N. 2013. Lista actualizada de las macroalgas marinas bentónicas de Argelia. I. Phaeophyceae. Anales Jard. Bot. Madrid 70(2): 136-143 (en inglés).

La diversidad de las algas marinas del Mediterráneo no es del todo conocida, especialmente en algunas áreas de su costa africana. Como parte de un esfuerzo para completar un catálogo más detallado, que permita reducir esta carencia, se aporta una lista crítica de las algas pardas (Phaeophyceae) de Argelia mediante la recopilación y actualización de todas las citas publicadas, tomando como punto de partida la de Perret-Boudouresque \& Seridi publicada el año 1989. Como resultado, el número total de táxones, a nivel específico e infraespecífico, aceptado para las costas de Argelia es de 93, de acuerdo con la taxonomía y la nomenclatura actuales.

Palabras clave: costa argelina, algas pardas, catálogo, Phaeophyceae, algas marinas.

\section{MATERIAL AND METHODS}

All brown algal taxa reported in the literature from Algeria have been searched, critically revised and treated according to current taxonomy and nomenclature in order to compile an updated list of the Algerian Phaeophyceae. Taxonomy and nomenclature follow Guiry \& Guiry (2013). In the catalogue, orders, genera and species were arranged alphabetically. When a taxon has been recorded under a synonym, this name is cited after the reference. For information included in unpublished works, locations are indicated after the authors' citations (Fig. 1). For each taxon the phytogeographic group is annotated according to Cormaci \& al. (1982) (A = Atlantic, $\mathrm{Ab}=$ Boreo-Atlantic, $\mathrm{Abt}=$ Boreo-tropical Atlantic, $\mathrm{AP}=\mathrm{At}-$ lanto-Pacific, $\mathrm{At}=$ Atlantic tropical, $\mathrm{C}=$ Cosmopolitan, $\mathrm{CB}=$ Circumboreal, $\mathrm{CBA}=$ Circumboreo-austral, $\mathrm{CT}=$ Circumtropical, IA = Indo-Atlantic, IAct = Indo-Atlantic cold temperate, $\mathrm{IP}=$ Indo-Pacific, $\mathrm{M}=$ Mediterranean, $\mathrm{SC}=$ Sub-cosmopolitan). For several species, comments on taxonomic, lifecycle stage or misidentifications are included. A list of taxa inquirenda as well as a list of taxa excludenda is also given.

\section{RESULTS}

\section{Cutleriales}

Cutleria adspersa (Mertens ex Roth) De Notaris. (CB)

Perret-Boudouresque \& Seridi, 1989; Seridi, 1990 (Surcouf); 


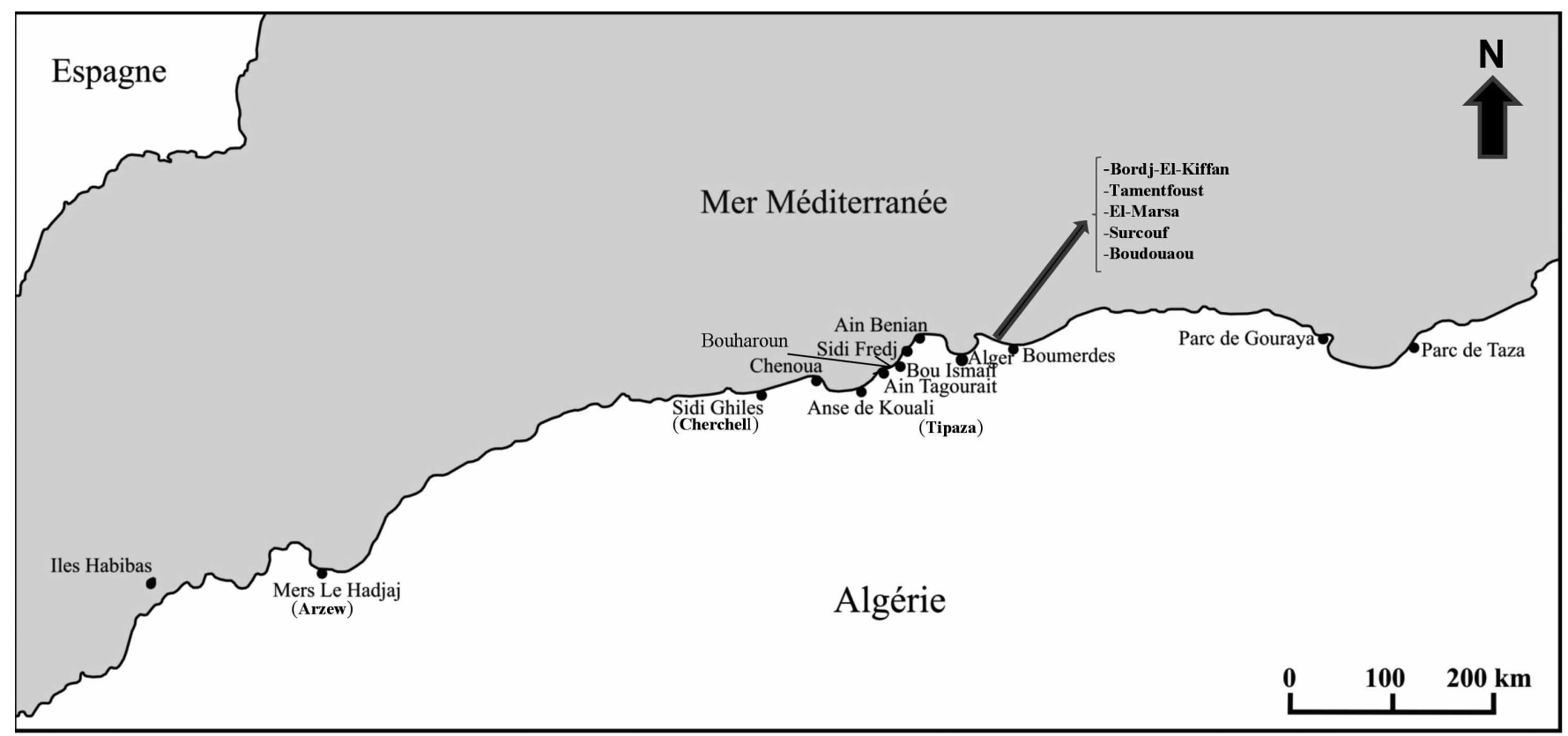

Fig. 1. Geographical distribution of the studied locations along the Algerian coast.

Ould-Ahmed, 1994 (Mers El Hadjadj), sporophyte not recorded; Ould-Ahmed, 2013 (Iles Habiba, Arzew, Gouraya, Taza), sporophyte not recorded.

This species includes also the sporophytic stage Aglaozonia melanoidea Schousboe ex Bornet.

Cutleria chilosa (Falkenberg) P.C. Silva (M)

Perret-Boudouresque \& Seridi, 1989, gametophyte not recorded.

This specific name is based on the sporophytic stage Aglaozonia chilosa Falkenberg. This species includes the gametophytic stage Cutleria monoica Olivier.

Cutleria multifida (Turner) Greville (SC).

Perret-Boudouresque \& Seridi, 1989; Ould-Ahmed, 1994 (Mers El Hadjadj), gametophyte not recorded; KadariMéziane, 1994 (Bou-Ismail), gametophyte not recorded; Ould-Ahmed, 2013 (Ain Tagourait, Ain Benian).

This species includes the sporophytic stage Aglaozonia parvula (Greville) Zanardini.

\section{Zanardinia typus (Nardo) P.C. Silva (AP)}

Perret-Boudouresque \& Seridi, 1989, as Zanardinia prototypus (J.V. Lamouroux) Montagne; Ould-Ahmed, 2013, as Zanardinia prototypus (J.V. Lamouroux) Montagne (Kouali, Gouraya, Taza).

\section{Dictyotales}

Dictyopteris polypodioides (A.P. De Candolle) J.V. Lamouroux $(\mathrm{C})$.

Perret-Boudouresque \& Seridi, 1989, as Dictyopteris membranacea (Stackhouse) Batters; Seridi, 1990, as Dictyopteris membranacea (Stackhouse) Batters (Bordj El Kiffan, Sur- couf); Ould-Ahmed, 1994, as Dictyopteris membranacea (Stackhouse) Batters (Mers El Hadjadj); Seridi \& al., 2007; Ould-Ahmed, 2013, as Dictyopteris membranacea (Stackhouse) Batters (Iles Habiba, Arzew, Cherchell, Chenoua, Ain Tagourait, Kouali, Sidi Fredj, Gouraya, Taza).

Nizamuddin (1981) described Dictyopteris tripolitana based on material collected from various locations of the Mediterranean Sea, including Algeria. Perret-Boudouresque \& Seridi (1989) included D. tripolitana in D. membranacea and pointed out in a note that the former species needed to be revised. In any case, $D$. tripolitana has been treated as questionable by Ribera \& al. (1992) and Cormaci \& al. (2012).

Dictyota dichotoma (Hudson) J.V. Lamouroux (C).

Perret-Boudouresque \& Seridi, 1989; Seridi, 1990 (El Marsa, Surcouf, Tipaza); Kadari-Méziane, 1994 (Bou-Ismail); OuldAhmed, 1994 (Mers El Hadjadj); Ould-Ahmed, 2013 (Iles Habiba, Arzew, Cherchell, Chenoua, Ain Tagourait, Kouali, Bou.Ismail, Sidi Fredj, Ain Benian, Gouraya, Taza).

Dictyota dichotoma var. intricata (C. Agardh) Greville (SC) Perret-Boudouresque \& Seridi, 1989.

Tronholm \& al. (2010) noted that this variety could not be distinguished from $D$. dichotoma using genetic markers applied in their study.

Dictyota fasciola (Roth) J.V. Lamouroux (SC).

Perret-Boudouresque \& Seridi, 1989, as Dilophus fasciola (Roth) M.A. Howe; Seridi, 1990, as Dilophus fasciola (Roth) M.A. Howe (Bordj El Kiffan, Tamentfoust, El Marsa, Surcouf, Sidi Fredj, Bouharoum); Kadari-Méziane, 1994, as Dilophus fasciola (Roth) M.A. Howe (Bou-Ismail); Seridi \& al., 2007; Ould-Ahmed, 2013, as Dilophus fasciola (Roth) M.A. Howe (Arzew, Gouraya). 
Dictyota fasciola var. repens (J. Agardh) Ardissone (IA).

Perret-Boudouresque \& Seridi, 1989; Ould-Ahmed, 1994, as Dilophus fasciola var. repens (J. Agardh) Feldmann (Mers El Hadjadj).

Dictyota implexa (Desfontaines) J.V. Lamouroux (SC).

Perret-Boudouresque \& Seridi, 1989, as Dictyota linearis (C. Agardh) Greville; Ould-Ahmed, 1994, as Dictyota linearis (C. Agardh) Greville (Mers El Hadjadj); Ould-Ahmed, 2013, as Dictyota linearis (C. Agardh) Greville (Iles Habiba, Arzew, Kouali, Bou-Ismail, Sidi Fredj, Ain Benian, Gouraya, Taza).

Dictyota spiralis Montagne (A).

Perret-Boudouresque \& Seridi, 1989, as Dilophus spiralis (Montagne) G. Hamel; Seridi, 1990, as Dilophus spiralis (Montagne) G. Hamel (Tamentfoust, Surcouf, Boudouaou, Bouharoum, Tipaza); Seridi \& al., 2007; Ould-Ahmed, 1994, as Dilophus spiralis (Montagne) G. Hamel (Mers El Hadjadj); Ould-Ahmed, 2013, as Dilophus spiralis (Montagne) G. Hamel (Iles Habiba, Arzew, Cherchell, Chenoua, Sidi Fredj, Ain Benian, Taza).

Lobophora variegata (J.V. Lamouroux) Womersley (SC) Perret-Boudouresque \& Seridi, 1989.

Padina pavonica (Linnaeus) Thivy (CT)

Perret-Boudouresque \& Seridi, 1989; Seridi, 1990; OuldAhmed, 1994 (Mers El Hadjadj); Kadari-Méziane, 1994 (BouIsmail); Seridi \& al., 2007; Ould-Ahmed, 2013 (Iles Habiba, Arzew, Cherchell, Chenoua, Ain Tagourait, Kouali, Bou-Ismail, Sidi Fredj, Ain Benian, Bourmedes, Gouraya, Taza).

Spatoglossum solieri (Chauvin ex Montagne) Kützing (IA) Perret-Boudouresque \& Seridi, 1989; Ould-Ahmed, 2013 (Iles Habiba, Gouraya, Taza).

Taonia atomaria (Woodward) J. Agardh (IA)

Perret-Boudouresque \& Seridi, 1989; Seridi, 1990 (El Marsa); Ould-Ahmed, 1994 (Mers El Hadjadj); Ould-Ahmed, 2013 (Arzew, Cherchell, Ain Tagourait, Bou-Ismail).

Zonaria tournefortii (J.V. Lamouroux) Montagne (IA)

Perret-Boudouresque \& Seridi, 1989; Ould-Ahmed, 2013 (Kouali, Gouraya, Taza).

\section{Ectocarpales}

Acinetospora crinita (Carmichael) Sauvageau (Abt)

Perret-Boudouresque \& Seridi, 1989, as Acinetospora vidovichii (Meneghini) Sauvageau.

\section{Asperococcus bullosus J.V. Lamouroux (SC)}

Perret-Boudouresque \& Seridi, 1989, as Asperococcus turneri (Smith) Hooker.

\section{Cladosiphon mediterraneus Kützing (M)}

Perret-Boudouresque \& Seridi, 1989.

Corynophlaea flaccida (C. Agardh) Kützing (M)

Perret-Boudouresque \& Seridi, 1989.

Colpomenia peregrina Sauvageau (SC)

Perret-Boudouresque \& Seridi, 1989; Ould-Ahmed, 2013 (Sidi Fredj).

Colpomenia sinuosa (Mertens ex Roth) Derbès \& Solier (C) Perret-Boudouresque \& Seridi, 1989; Seridi, 1990 (Tamentfoust, Surcouf, Boudouaou); Ould-Ahmed, 1994 (Mers El Hadjadj); Seridi \& al., 2007; Ould-Ahmed, 2013 (Iles Habiba, Arzew, Cherchell, Chenoua, Ain Tagourait, Kouali, BouIsmail, Sidi Fredj, Ain Benian, Gouraya, Taza).

Ectocarpus fasciculatus Harvey $(\mathrm{Ab})$

Perret-Boudouresque \& Seridi, 1989; Ould-Ahmed, 2013 (Chenoua).

Ectocarpus siliculosus (Dillwyn) Lyngbye (C)

Perret-Boudouresque \& Seridi, 1989; Kadari-Méziane, 1994, as Ectocarpus siliculosus var. confervoides Le Jolis (Bou-Ismail); Ould-Ahmed, 1994, as Ectocarpus confervoides Le Jolis (Mers El Hadjadj); Ould-Ahmed, 2013, as Ectocarpus confervoides Le Jolis (Iles Habiba, Arzew, Chenoua, Kouali, Ain Benian, Gouraya, Taza).

Elachista intermedia P.L. Crouan \& H.M. Crouan (Ab)

Perret-Boudouresque \& Seridi, 1989; Ould-Ahmed, 2013 (Kouali).

Feldmannia caespitula (J. Agardh) Knoepffler-Péguy (IAct) Perret-Boudouresque \& Seridi, 1989; Ould-Ahmed, 2013 (Kouali, Ain Benian).

Feldmannia levelii (Areschoug ex P.L. Crouan \& H.M. Crouan) G. Hamel (Ab)

Perret-Boudouresque \& Seridi, 1989, as Feldmannia caespitula var. levelii (Areschoug ex P.L. Crouan \& H.M. Crouan) Knoepffler-Péguy.

Feldmannia globifera (Kützing) G. Hamel (SC)

Perret-Boudouresque \& Seridi, 1989.

According to Cormaci \& al. (2012), this species is synonymous with Feldmannia paradoxa (Montagne) Hamel.

Feldmannia irregularis (Kützing) G. Hamel (C)

Perret-Boudouresque \& Seridi, 1989; Ould-Ahmed, 2013 (Kouali).

Feldmannia simplex (P.L. Crouan \& H.M. Crouan) G. Hamel (SC)

Perret-Boudouresque \& Seridi, 1989.

According to Cormaci \& al. (2012), this species is synony- 
mous with Feldmannia paradoxa var. caespitula (J. Agardh) Cormaci \& G. Furnari.

Giraudia sphacelarioides Derbès \& Solier (IAct)

Perret-Boudouresque \& Seridi, 1989; Ould-Ahmed, 2013 (Kouali).

Hincksia mitchelliae (Harvey) P.C. Silva (C)

Perret-Boudouresque \& Seridi, 1989, as Giffordia mitchelliae (Harvey) Hamel.

Hincksia sandriana (Zanardini) P.C. Silva (SC)

Perret-Boudouresque \& Seridi, 1989, as Giffordia sandriana Zanardini) Hamel.

Hincksia secunda (Kützing) P.C. Silva (IAct)

Perret-Boudouresque \& Seridi, 1989, as Giffordia secunda (Kützing) Batters; Ould-Ahmed, 2013, as Giffordia secunda (Kützing) Batters (Kouali).

Hydroclathrus clathratus (Bory ex C. Agardh) Howe (C) Perret-Boudouresque \& Seridi, 1989.

Kuckuckia spinosa (Kützing) Kornmann (Ab)

Kadari- Méziane (Bou-Ismail); Perret-Boudouresque \& Seridi, 1989; Ould-Ahmed, 1994 (Mers El Hadjadj); OuldAhmed, 2013 (Arzew, Kouali).

Kuetzingiella battersi (Bornet ex Sauvageau) Kormann (SC) Perret-Boudouresque \& Seridi, 1989.

Kuetzingiella battersi var. mediterranea Gómez \& Ribera (M)

Perret-Boudouresque \& Seridi, 1989.

Although this taxon was not on the species list by PerretBoudouresque \& Seridi (1989), as indicated by Ribera \& al. (1992), it was included in the citations of Kuetzingiella battersi.

Liebmannia leveillei J. Agardh (Abt)

Perret-Boudouresque \& Seridi, 1989; Ould-Ahmed, 2013

(Taza).

Myriactula arabica (Kützing) Feldmann (IP)

Perret-Boudouresque \& Seridi, 1989.

Myriactula gracilariae Feldmann (M)

Perret-Boudouresque \& Seridi, 1989; Ould-Ahmed, 2013 (Kouali).

The record of this species by Ould-Ahmed (2013) is the second citation, since Cormaci \& al. (2012) indicated that this species was not recorded ever again after its description.

Myriactula rigida (Sauvageau) G. Hamel (M)

Perret-Boudouresque \& Seridi, 1989.
Myriactula rivulariae (Suhr ex Areschoug) Feldmann (IA)

Perret-Boudouresque \& Seridi, 1989.

Myrionema orbiculare J. Agardh (SC)

Perret-Boudouresque \& Seridi, 1989; Ould-Ahmed, 2013 (Kouali).

Myrionema strangulans Greville (SC)

Perret-Boudouresque \& Seridi, 1989; Ould-Ahmed, 2013 (Kouali).

Myriotrichia claviformis Harvey $(\mathrm{Ab})$

Perret-Boudouresque \& Seridi, 1989, as Streblonema sphaericum (Derbès \& Solier) Thuret.

Myriotrichia repens Hauck (A)

Ould-Ahmed, 2013 (Kouali).

Nemacystus flexuosus (C. Agardh) Kylin var. giraudyi (J. Agardh) De Jong (M)

Ould-Ahmed, 2013, as Nemacystus ramulosus Derbès \& Solier (Kouali).

Nemacystus hispanicus (Sauvageau) Kylin (A)

Perret-Boudouresque \& Seridi, 1989.

Petalonia fascia (O.F. Müller) Kuntze (C)

Perret-Boudouresque \& Seridi, 1989; Seridi, 1990 (Bordj El Kiffan, Surcouf, Boudouaou); Kadari-Méziane, 1994 (BouIsmail); Ould-Ahmed, 2013 (Bou-Ismail, Ain Benian).

Punctaria latifolia Greville (CBA)

Perret-Boudouresque \& Seridi, 1989.

Rosenvingea intricata (J. Agardh) Boergesen (C)

Perret-Boudouresque \& Seridi, 1989.

Sauvageaugloia divaricata (Clemente) Cremades (IA)

Perret-Boudouresque \& Seridi, 1989, as Sauvageangloia griffithsiana (Greville) G. Hamel ex Kylin.

Scytosiphon lomentaria (Lyngbye) Link (C)

Perret-Boudouresque \& Seridi, 1989; Seridi, 1990 (Bouduaou); Ould-Ahmed, 1994 (Mers El Hadjadj); OuldAhmed, 2013 (Arzew, Chenoua, Kouali, Sidi Fredj).

Spermatochnus paradoxus (Roth) Kützing ( $\mathrm{Ab}$ )

Perret-Boudouresque \& Seridi, 1989.

Stragularia clavata (Harvey) G. Hamel (C)

Kadari-Méziane, 1994 (Bou-Ismail).

According to Cormaci \& al. (2012), this taxon corresponds to the sporophyte of Petalonia fascia.

Zosterocarpus oedogonium (Meneghini) Bornet (M) 
Perret-Boudouresque \& Seridi, 1989.

\section{Fucales}

Cystoseira algeriensis Feldmann (M)

Perret-Boudouresque \& Seridi, 1989; Ould-Ahmed, 2013 (Ain Benian).

Cystoseira amentacea var. stricta Montagne (M)

Perret-Boudouresque \& Seridi, 1989, as Cystoseira stricta (Montagne) Sauvageau; Seridi, 1990, as Cystoseira stricta (Montagne) Sauvageau (Boudouau); Ould-Ahmed, 1994, as Cystoseira stricta (Montagne) Sauvageau (Mers El Hadjadj); Kadari-Méziane, 1994 (Bou-Ismail); Seridi \& al., 2007; OuldAhmed, 2013 (Iles Habiba, Arzew, Cherchell, Chenoua, Ain Tagourait, Ain Benian, Bourmedes, Gouraya, Taza).

Cystoseira barbata (Stackhouse) C. Agardh (M)

Ould-Ahmed, 2013 (Cherchell, Ain Tagourait, Kouali).

Perret-Boudouresque \& Seridi (1989) placed this species in the taxa excludenda list, according to Sauvageau (1912), who pointed out that he did not know any herbarium specimen from the Mediterranean coasts of Africa. Provided that this species has been reported from many places on these coasts, such as Egypt (Aleem, 1993) and Libya (Ribera \& al., 1992), we accept it as present in Algeria as well.

Cystoseira brachycarpa J. Agardh (M)

Perret-Boudouresque \& Seridi, 1989, as Cystoseira caespistosa Sauvageau; Ould-Ahmed, 2013, as Cystoseira caespistosa Sauvageau (Ain Benian).

Cystoseira compressa (Esper) Gerloff \& Nizamuddin (Ab) Perret-Boudouresque \& Seridi, 1989; Seridi, 1990 (El Marsa, Surcouf, Boudouaou, Tipaza); Ould-Ahmed, 1994 (Mers El Hadjadj); Seridi \& al., 2007; Kadari-Méziane, 1994 (Bou-Ismail); Ould-Ahmed, 2013 (Iles Habiba, Arzew, Cherchell, Chenoua, Ain Tagourait, Kouali, Bou-Ismail, Sidi Fredj, Ain Benian, Bourmedes, Taza).

\section{Cystoseira crinita Duby (M)}

Perret-Boudouresque \& Seridi, 1989; Ould-Ahmed, 1994 (Mers El Hadjadj); Ould-Ahmed, 2013 (Iles Habiba, Arzew, Cherchell, Sidi Fredj, Taza).

\section{Cystoseira foeniculacea (Linnaeus) Greville (M)}

Perret-Boudouresque \& Seridi, 1989, as Cystoseira ercegovicii Giaccone; Seridi, 1990, as Cystoseira ercegovicii Giaccone (Surcouf, Bouharoum); Ould-Ahmed, 1994, as Cystoseira ercegovicii Giaccone (Mers El Hadjadj); Ould-Ahmed, 2013, as Cystoseira ercegovicii Giaccone (Arzew, Bourmedes, Taza).

Cystoseira humilis Schousboe ex Kützing ( $\mathrm{Ab}$ )

Ould-Ahmed, 2013 (Gouraya).

Cystoseira mediterranea Sauvageau (M)

Seridi, 1990 (Bordj El Kiffan, Sidi Fredj, Bouharoum).
Cystoseira sauvageauana Hamel (M)

Perret-Boudouresque \& Seridi, 1989; Ould-Ahmed, 2013 (Cherchell, Kouali).

Cystoseira sedoides (Desfontaines) C. Agardh (M)

Perret-Boudouresque \& Seridi, 1989; Seridi, 1990 (Surcouf); Ould-Ahmed, 2013 (Cherchell, Chenoua, Kouali, Gouraya).

Cystoseira spinosa Sauvageau (M)

Perret-Boudouresque \& Seridi, 1989; Ould-Ahmed, 2013 (Gouraya, Taza).

Cystoseira tamariscifolia (Hudson) Papenfuss (Ab)

Perret-Boudouresque \& Seridi, 1989; Ould-Ahmed, 1994 (Mers El Hadjadj); Ould-Ahmed, 2013 (Iles Habiba, Arzew, Cherchell, Chenoua, Ain Tagourait, Kouali, Sidi Fredj, Ain Benian, Bourmedes, Gouraya, Taza).

Cystoseira usneoides (Linnaeus) M. Roberts ( $\mathrm{Ab}$ )

Perret-Boudouresque \& Seridi, 1989; Ould-Ahmed, 2013 (Gouraya).

Cystoseira zosteroides (Turner) C. Agardh (M)

Perret-Boudouresque \& Seridi, 1989.

Sargassum acinarium (Linnaeus) Setchell (CT)

Perret-Boudouresque \& Seridi, 1989, as Sargassum acinarium (Linnaeus) C. Agardh.

Sargassum hornschuchii C. Agardh (M)

Perret-Boudouresque \& Seridi, 1989; Ould-Ahmed, 2013 (Gouraya).

Sargassum trichocarpum J. Agardh (M)

Perret-Boudouresque \& Seridi, 1989.

Sargassum vulgare C. Agardh (CT)

Perret-Boudouresque \& Seridi, 1989; Seridi, 1990 (Bordj El Kiffan, Tamentfoust, Surcouf, Sidi Fredj); Kadari-Méziane, 1994 (Bou-Ismail); Ould-Ahmed, 1994 (Mers El Hadjadj); Seridi \& al., 2007; Ould-Ahmed, 2013 (Arzew, Cherchell, Ain Tagourait, Kouali, Bou-Ismail, Sidi Fredj, Ain Benian, Bourmedes, Gouraya, Taza).

\section{Laminariales}

Laminaria ochroleuca Bachelot de la Pylaie (A)

Perret-Boudouresque \& Seridi, 1989.

Laminaria rodriguezii Bornet $(\mathrm{M})$

Perret-Boudouresque \& Seridi, 1989.

Phyllariopsis brevipes (C. Agardh) E.C. Henry \& G.R. South $(\mathrm{Ab})$

Perret-Boudouresque \& Seridi, 1989; Ould-Ahmed, 2013 (Taza). 


\section{Nemodermatales}

Nemoderma tingitanum Schousboe ex Bornet (At)

Perret-Boudouresque \& Seridi, 1989.

\section{Ralfsiales}

Hapalospongidium macrocarpum (Feldmann) León-Álvarez \& González-González (M)

Perret-Boudouresque \& Seridi, 1989, as Mesospora macrocarpa (Feldmann) den Hartog; Kadari-Méziane, 1994, as Mesospora macrocarpa (Feldmann) den Hartog (Bou-Ismail).

Pseudolithoderma adriaticum (Hauck) Verlaque (M)

Perret-Boudouresque \& Seridi, 1989.

Ralfsia verrucosa (Areschoug) Areschoug (SC)

Perret-Boudouresque \& Seridi, 1989; Kadari-Méziane, 1994 (Bou-Ismail); Ould-Ahmed, 2013 (Chenoua, Kouali, Bou-Ismail, Taza).

\section{Scytothamnales}

Bachelotia antillarum (Grunow) Gerlof (C)

Ould-Ahmed, 2013, as Bachelotia fulvescens (Bornet) Kuckuck ex G. Hamel (Kouali).

The presence of this species in the Mediterranean Sea needs to be confirmed. Cormaci \& al. (2012) excluded this taxon from the Adriatic Sea. This is due to the fact that, in reporting this species (as Pylaiella fulvescens Bornet), Schiffner \& Vatova (1937) stated that they only provisionally attributed their specimens to this species, while they very probably belonged to an undescribed taxon.

\section{Sphacelariales}

Cladostephus spongiosus (Hudson) C. Agardh (IA)

Perret-Boudouresque \& Seridi, 1989, as Cladostephus hirsutus (Linnaeus) Boudouresque \& Perret; Ould-Ahmed, 2013, as Cladostephus hirsutus (Linnaeus) Boudouresque \& PerretBoudouresque (Kouali, Bou-Ismail, Ain Benian, Gouraya, Taza).

\section{Halopteris filicina (Grateloup) Kützing (SC)}

Perret-Boudouresque \& Seridi, 1989; Seridi, 1990 (Surcouf, Tipaza); Kadari-Méziane, 1994 (Bou-Ismail); Ould-Ahmed, 1994 (Mers El Hadjadj); Ould-Ahmed, 2013 (Iles Habiba, Arzew, Cherchell, Chenoua, Ain Tagourait, Kouali, Sidi Fredj, Ain Benian, Gouraya, Taza).

Sphacelaria cirrosa (Roth) C. Agardh (SC)

Perret-Boudouresque \& Seridi, 1989; Kadari-Méziane, 1994 (Bou-Ismail); Ould-Ahmed, 1994 (Mers El Hadjadj); Seridi \& al., 2007; Ould-Ahmed, 2013 (Iles Habiba, Arzew, Kouali, Sidi Fredj, Ain Benian, Taza).

Sphacelaria rigidula Kützing (C)

Perret-Boudouresque \& Seridi, 1989; Ould-Ahmed, 2013, as
Sphacelaria furcigera Kützing (Iles Habiba, Kouali).

Sphacelaria plumula Zanardini $(\mathrm{Ab})$

Kadari-Méziane, 1994 (Bou-Ismail); Ould-Ahmed, 2013 (Iles Habiba, Kouali, Taza).

\section{Sphacelaria tribuloides Meneghini (C)}

Perret-Boudouresque \& Seridi, 1989; Seridi, 1990 (Surcouf); Kadari-Méziane, 1994 (Bou-Ismail); Ould-Ahmed, 1994 (Mers El Hadjadj); Ould-Ahmed, 2013 (Iles Habiba, Arzew, Chenoua, Ain Benian, Gouraya, Taza).

Stypocaulon scoparium (Linnaeus) Kützing (SC)

Perret-Boudouresque \& Seridi, 1989; Seridi, 1990 (Tamentfoust, Surcouf, Bouharoum, Tipaza); Kadari-Méziane, 1994 (Bou-Ismail); Ould-Ahmed, 1994 (Mers El Hadjadj); Seridi \& al., 2007, as Halopteris scoparia (Linnaeus) Sauvageau; Ould-Ahmed, 2013 (Iles Habiba, Arzew, Cherchell, Ain Tagourait, Kouali, Bou-Ismail, Sidi Fredj, Ain Benian, Gouraya).

\section{Sporochnales}

Nereia filiformis (J. Agardh) Zanardini (At)

Perret-Boudouresque \& Seridi, 1989; Ould-Ahmed, 2013 (Iles Habiba).

\section{Tilopteridales}

Climacosorus mediterraneus Sauvageau (M)

Perret-Boudouresque \& Seridi, 1989.

\section{Taxa inquirenda}

Cystoseira montagnei J. Agardh

Perret-Boudouresque \& Seridi, 1989; Ould-Ahmed, 2013 (Taza).

The taxonomic position of this taxon is uncertain and its status remains obscure in the Mediterranean Sea (Cormaci \& al., 2012). Ribera \& al. (1992) included the records of this species in their taxa inquirenda list.

Cystoseira platyclada Sauvageau

Perret-Boudouresque \& Seridi, 1989.

Ribera \& al. (1992) included these records in their taxa inquirenda list.

\section{Taxa excludenda}

Cystoseira sauvageauna var. polyodematis (Sauvageau) Hamel

Perret-Boudouresque \& Seridi, 1989

According to Motta (1989), this taxon does not differ from the type variety. Ribera \& al. (1992) included this variety in their taxa excludenda list.

Ectocarpus commensalis Setchell \& N.L. Gardner 
Perret-Boudouresque \& Seridi, 1989, as Ectocarpus parvus (De A. Saunders) Hollenberg; Seridi, 1990, as Ectocarpus parvus (De A. Saunders) Hollenberg (Surcouf); OuldAhmed, 2013, as Ectocarpus parvus (De A. Saunders) Hollenberg (Iles Habiba, Kouali, Ain Benian).

According to Cormaci \& al. (2012), this species should be excluded from the Mediterranean algal flora.

\section{DISCUSSION}

The present checklist encompasses 93 accepted brown algal taxa registered to date in Algeria, 8 more than those included in Perret-Boudouresque \& Seridi (1989). In addition, two taxa are considered as taxa inquirenda and two as taxa excludenda. In the great part of the Mediterranean countries, modern studies have enormously increased the known number of taxa of the flora of seaweeds. On the other hand, we have not found in Algeria many new records, perhaps because this flora had already been studied in depth by classical authors as stated in the introduction.

Concerning the 8 new species cited in Algeria, three of them (Bachelotia antillarum, Myriotrichia repens and Nemacystus flexuosus) are small species epiphytes on Posidonia oceanica (L.) Delile leaves, three species are Fucales belonging to Cystoseira genus (Cystoseira barbata, Cystoseira humilis and Cystoseira mediterranea), and the remaining two are Sphacelaria plumula and Stragularia clavata.

Bachelotia antillarum is a sub-cosmopolitan taxa cited previously only in the Mediterranean Sea by Schiffner \& Vatova (1937) and Vatova (1940) from Venice Lagoon as Pylaiella fulvescens and based on the above papers reported by Furnari \& al. (2010) from the Adriatic Sea. However, as indicated, Cormaci \& al. (2012) excluded this taxon from the Adriatic Sea. We consider that the presence of this species in the Mediterranean Sea needs to be confirmed. Myriotrichia repens and Nemacystus flexuosus have been found only once by Ould-Ahmed (2013) on the Algerian coast, but both species are known on the African Mediterranean coasts in Tunisia (Ribera \& al., 1992, the former species given erroneously as Myriotrichia clavaeformis Harvey).

Cystoseira barbata has been found by Ould-Ahmed (2013) in three locations of the Tipaza region. Although PerretBoudouresque \& Seridi (1989) included this species among the taxa excludenda, its presence was already cited by Ribera \& al. (1992) on the nearby coasts of Egypt, Libya and Tunisia. Cystoseira humilis has been cited by Ould-Ahmed (2013) in the National Park of Gouraya; this is the first citation for the African Mediterranean coasts since the citation by Báez \& al. $(2005 \mathrm{a}, \mathrm{b})$ is erroneous. This is because these authors used as reference Ribera \& al. (2012), who did not cite this species in this area. Cystoseira mediterranea was cited by Seridi (1990) in three locations from the center of Algeria coasts and was previously cited by Ribera \& al. (1992) in Tunisia and on the Mediterranean coast of Morocco.

Sphacelaria plumula was found by Kadari-Mèziane (1994) in the area of Bou-Ismail and by Ould-Ahmed (2013) in several locations along the coasts of Algeria; this species was cited in Libya and Tunisia by Ribera \& al. (1992). Stragularia clavata was cited by Kadari-Mèziane (1994) in the area of
Bou-Ismail. This species was known from the North and East of the Mediterranean Sea but not from the African Mediterranean coasts (Ribera \& al., 1992, Guiry \& Guiry, 2013).

The most cited taxa in Algeria by different authors and in many places on theses coasts belong to Fucales (Cystoseira amentacea var. stricta, Cystoseira compressa, Cystoseira tamariscifolia and Sargassum vulgare), Dictyotales (Dictyopteris polypodioides, Dictyota dichotoma, Dictyota fasciola, Dictyota spiralis and Padina pavonica) and Sphacelariales (Halopteris filicina, Stypocaulon scoparium and Colpomenia sinuosa). On the other hand, some taxa often cited by classical authors in many places on the central coasts of Algeria are rarely cited in recent papers. This is the case of Cystoseira sauvageauana only cited in two places (Cherchell and Kouali) by Ould-Ahmed (2013), and of Phyllariopsis brevipes only found in the Natural Park of Taza (Ould-Ahmed, 2013). In the case of the latter species, the lack of recent citations could be due to the subtidal habitat of the species, a habitat that is not usually studied. Even other subtidal species previously cited by classical authors have not been found recently, such as Laminaria ochroleuca, Laminaria rodriguezii, Sargassum acinarium, Sargassum hornschuchii and Sargassum trichocarpum.

Colpomenia peregrina is the sole Algerian Phaeophyceae included in the list of introduced species of the Mediterranean Sea (Zenetos \& al., 2010, 2012). This species has an Indo-Pacific origin and has been found one meter deep in a Caulerpa racemosa (Forsskål) J. Agardh meadow (OuldAhmed, 2013).

In conclusion, the number of Phaeophyceae registered on the Algerian coasts is low in comparison with its total number for the entire Mediterranean Sea (214 taxa, Furnari \& al., 2010), or for the Adriatic Sea (180 taxa, Antolic \& al., 2010). However, it is similar to other Mediterranean countries such as Greece (107 taxa, Tsiamis \& al., 2013) and Tunisia (91 taxa, Guiry \& Guiry, 2013), and higher than Libya (53 taxa, Guiry \& Guiry, 2013).

Biogeographically, the Algerian flora of Phaeophyceae is characterized by a high number of Cosmopolitan or Sub-cosmopolitan taxa (31 taxa, 37\%), followed by the Mediterranean (25 taxa, 30\%), the Atlantic (20 taxa, 24\%) and the Indo-Atlantic (9 taxa, 11\%). The Circumboreal and Circumtropical taxa are scarcely represented (2 taxa each, $2 \%$ ). Concerning the Mediterranean taxa, almost half correspond to the Sargassaceae family, which make up the most characteristic communities of vegetation in the Mediterranean Sea.

\section{ACKNOWLEDGEMENTS}

The authors thank the support given by Professors Jordi Rull and Joan Vallès and by Joana Aragay, Antonio Gómez, and Samy Ould Ahmed. We are grateful to Tanya G. Johnson for reviewing the English text.

\section{REFERENCES}

Aleem, A.A. 1993. Marine algae of Alexandria, Egypt. Alexandria.

Antoli , A., Špan, A., Žuljevi , A., Nikoli , V., Grubeli , I., Despatalovi , M. \& Cvitkovi, I. (2010). A checklist of the benthic marine macroalgae from the eastern Adriatic coast: II. Heterokontophyta: Phaeophyceae. Acta Adriatica 51: 9-33.

Báez, J.C., Real, R., Vargas, J.M. \& Flores-Moya, A. 2004. A biogeographical analysis of the genera Audouinella (Rhodophyta), Cystoseira (Phaeophy- 
ceae) and Cladophora (Chlorophyta) in the western Mediterranean Sea and Adriatic Sea. Phycologia 43: 404-415.

Báez, J.C., Real, R., Vargas, J.M. \& Flores-Moya, A. 2005a. Chorotypes of seaweeds from the western Mediterranean Sea and the Adriatic Sea: An analysis based on the genera Audouinella (Rhodophyta), Cystoseira (Phaeophyceae) and Cladophora (Chlorophyta). Phycological Research 53: 255-265.

Báez, J.C., Olivero, J., Real, R., Vargas, J.M. \& Flores-Moya, A. 2005b. Analysis of geographical variation in species richness within the genera Audouinella (Rhodophyta), Cystoseira (Phaeophyceae) and Cladophora (Chlorophyta) in the western Mediterranean Sea. Botanica Marina 48: 30-37.

Cormaci, M., Duro, A. \& Furnari, G. 1982. Considerazioni sugli elemti fitogeografici della flora algale della Sicilia. Naturalista Siciliano, Ser. IV 6 (suppl.): 7-14.

Cormaci, M., Furnari, G., Catra, M., Alongi, G. \& Giaccone, G. 2012. Flora marina bentonica del Mediterraneo: Phaeophyceae. Bollettino dell'Accademia Gioenia di Scienze Naturali di Catania 45: 1-508.

Debray, F. 1893. Liste des algues marines et d'eau douce récoltées jusqu'à ce jour en Algérie. Bull.sci. Fr. Belg. 25: 1-19.

Debray, F. 1897. Catalogue des algues du Maroc, d'Algérie \& de Tunisie. Librairie Adolphe Jourdan Edit. Alger.

Furnari, G., Giaccone, G., Cormaci, M., Alongi, G., Catra, M., Nisi, A. \& Serio, D. 2010. Macrophytobenthos. In: Relini, G. Checklist della flora e della fauna dei mari italiani (parte II). Biologia Marina Mediterranea 17(Suppl. 1): 801-828.

Guiry, M.D. \& Guiry, G.M. 2013. AlgaeBase. World-wide electronic publication, National University of Ireland, Galway. http://www.algaebase.org; searched on 27 February 2013.

Kadari-Méziane, Y., 1994. Contribution à l'étude de l'impact de la pollution sur la distribution spatio-temporelle des peuplements phytobenthiques dans la baie de Bou-Ismail (Algérie). Thèse Magister ENS, Alger.

Montagne, C., 1838. Cryptogames algériennes, ou plantes cellulaires recueillies par M. Roussel aux environs d'Alger, et publiées par le Docteur Camille Montagne. Annales des Sciences Naturelles, Botanique, série 210 : 268-279.

Montagne, C. 1846-1849. Ordo I. Phyceae Fries. In: Bory de Saint-Vincent et Durieu De Maisonneuve. Exploration scientifique de l'Algérie.

Montagne, C. 1856. Sylloge generum specierumque cryptogamarum quas in variis operibus descriptas iconobusque illustratas, nunc ad diagnosim reductas, nonnullasque novas interjectas, ordine systematijco disposuit. J.B. Baillieres Edit., Paris.

Motta, G., 1989. Fenologia morfologica e riproduttiva di Cystoseria sauvageauana Hamel (Phaeophyta, Fucales). Bollettino dell'Accademia Gioenia di Scienze Naturali di Catania 22:317-334.

Nizamuddin, M. 1981. Contribution to the marine algae of Libya Dictyotales. Bibliotheca Phycologica 54: 1-122.

Ould-Ahmed, N., 1994. Étude des espèces phytobenthiques au voisinage de la centrale thermique de Mers El Hadjdj (Golfe d'Arzew; Ouest algérien). Mention particulière sur une espèce remarquable Chlorophyte, Caulerpale: Caulerpa prolifera (Forsskal) Lamouroux. Thèse Magister, ISMAL. Alger.

Ould-Ahmed, N., 2005. Note sur les algues des côtes algériennes. INOCCOMSTECH-ISESCO. The Protection of Costal and Environnement: 55-60.

Ould-Ahmed, N. 2013. Les algues des côtes algériennes. Connaissance, Caractérisation, Conservation et Utilisation. Thèse Doctorat (en cours de finalisation).

Ould-Ahmed, N., Bounaga, N. \& Cirik, C. 1995. Le peuplement algal au voisinage de la centrale thermique de Mers-El Hadjadj (Golfe d'Arzew; ouest algérien): Affinités biogéographiques. Rapport Commission International Mer Méditerranée (Malte) 34:38.

Ould-Ahmed, N. \& Meinesz, A. 1998. Note sur la prédominance d'une Chlorophyte Caulerpale. Caulerpa prolifera (Forsskal) Lamouroux, au voisinage d'une centrale à production d'électricité (804 M.W.) de MersEl-Hadjadj (Golfe d'Arzew; Ouest algérien). Rapport Commission International Mer Méditerranée (Croatie). 35(2): 566-567.

Ould-Ahmed, N. \& Meinesz, A. 2007. First record of the invasive alga Caulerpa racemosa (Caulerpales, Chlorophyta) on the coast of Algeria. Cryptogamie Algologie 28: 303-305.

Perret-Boudouresque, M. \& Seridi, H. 1989. Inventaire des algues marines benthiques d'Algerie. GIS Posidonie publ., Marseille. 1-117.

Ribera, M.A., Gómez-Garreta, A., Gallardo, T., Cormaci, M., Furnari, G. \& Giaccone, G. 1992. Check-list of Mediterranean Seaweeds. I. Fucophyceae (Warming 1884). Botanica Marina 35: 109-130.

Sauvageau, C. (1912). À propos des Cystoseira de Banyuls et Guéthary. Bulletin Station Biologique Arcachon 14: 133-556.

Schiffner, V. \& Vatova, A. 1937. Le alghe della laguna Veneta. In: La laguna di Venezia. Vol. $3\left(5^{\circ}, 9\right)$, Ferrari Eds.

Seridi, H., 1990. Étude des algues marines benthiques de la région d'Alger. Thèse Magister USTHB. Alger.

Seridi, H., 2007. Étude de la flore algale de l'Algérie. Etude phytosociologique des peuplements algaux photophiles de l'infralittoral supérieur de substrat dur. Thèse Doctorat d'état és-Sciences Biologiques. USTHB, Alger.

Seridi, H., Ruitton, S. \& Boudouresque, Ch.F. 2007. Is it possible to calibrate the pollution level of the region of Algiers (Mediterranean Sea) by exploiting marine macrophytes? Comptes Rendus Biologies 330: 606-614.

Tronholm, A., Steen, F., Tyberghein, L., Leliaert, F. Verbruggen, H., Ribera Siguan, M.A. \& De Clerck, O. 2010. Species delimitation, taxonomy, and biogeography of Dictyota in Europe (Dictyotales, Phaeophyceae). Journal of Phycology 46: 1301-1321.

Tsiamis, K., Panayotidis, P., Economou-Amilli, A. \& Katsaros, C. 2013. Seaweeds of the Greek coasts. Phaeophyceae. Mediterranean Marine Science 14: 141-157.

Vatova, A. 1940. Distribuzione geografica delle alghe nella Laguna veneta e fattori che la determinano. Thalassia 4: 1-37.

Zenetos, A., Gofas, S., Verlaque, M., Cinar, M.E., García Raso, J.E., Bianchi, C.N., Morri, C., Azzurro, E., Bilecenoglu, M., Froglia, C., Siokou, I., Violanti, D., Sfriso, A., San Martin, G., Giangrande, A., Katagan, T., Ballesteros, E., Ramos-Espla, A., Mastrototaro, F., Ocaña, O., Zingone, A., Gambi, M.C. \& Streftaris, N., 2010. Alien species in the Mediterranean Sea by 2010. A contribution to the application of European Union's Marine Strategy Framework Directive (MSFD). Part I. Spatial distribution. Mediterranean Marine Science 11/2: 381-493.

Zenetos, A., Gofas, S., Morri, C., Rosso, A., Violanti, D., García Raso, J.E., Cinar, M.E., Almogi-Labin, A., Ates, A.S., Azzurro, E., Ballesteros, E., Bianchi, C.N., Bilecenoglu, M., Gambi, M.C., Giangrande, A., Gravili, C., Hyams-Kaphzan, O., Karachle, P.K., Katsanevakis, S., Lipe, L., Mastrototaro, F., Mineur, F., Pancucci-Papadopoulou, M.A., Ramos-Esplá, A., Salas, C., San Martin, G., Sfriso, A., Streftaris, N. \& Verlaque, M. 2012. Alien species in the Mediterranean Sea by 2012. A contribution to the application of European Union's Marine Strategy Framework Directive (MSFD). Mediterranean Marine Science 13/2:328-352.

Associate Editor: Javier Fuertes Received: 5-IV-2013 Accepted: 5-III-2014 